\title{
Kurzzeitige Veränderungen hydrographischer Faktoren und der Sestonkomponenten in driftenden Wassermassen in der Helgoländer Bucht*
}

\author{
W. HICKEL \\ Biologische Anstalt Helgoland (Zentrale); Hamburg, Bundesrepublik Deutschland
}

\begin{abstract}
Short-term variations of hydrographical factors and seston components in drifting water masses in Helgoland Bight. In Helgoland Bight (Inner German Bight, North Sea) drifting water masses were investigetad at 3 stations for 4-8 hours during August and November, 1968. Measurments of salinity, temperature, turbidity and seston weight were made and phytoplankton was counted in vertical series of water samples taken at about 30 - minute intervals. Only the water at drifting station "Außeneider" remained hydrographically unchanged during the investigation. The concentration of non-motile phytoplankton (diatoms) did not decrease with the slowing down of tidal current, whereas seston weight and turbidity decreased rapidly. Drift-cross tracking of the water at Helgoland Roads revealed that the water masses may circle around the island several times. Published results on horizontal diffusion and the present data indicate that measurements of changes within a plankton population over several days in Helgoland Bight require elaborate teamwork.
\end{abstract}

\section{EINLEITUNG}

Die Innere Deutsche Bucht (Helgoländer Bucht) zeichnet sich durch besonders unruhige hydrographische Verhältnisse aus. Gezeitenstrom-Geschwindigkeiten bis zu $3 \mathrm{sm} / \mathrm{h}$ in Küstennähe und $2 \mathrm{sm} / \mathrm{h}$ bei Helgoland werden regelmäßig erreicht und versetzen die Wassermassen um etwa 5 bis $10 \mathrm{sm}$ während einer halben Tide. Demgegenüber ist der Reststrom mit maximal $0,2 \mathrm{sm} / \mathrm{h}$ gering. Windeinwirkungen führen zu erheblichen Wasserstands- und Strömungsschwankungen. In Küstennähe bilden sich unterschiedliche Wassermassen durch den Zustrom von Elbe- und Weserwasser sowie durch den Einfluß ausgedehnter Wattgebiete.

Die Vielfalt und Veränderlichkeit der Wassermassen begrenzen die Möglichkeit der Messung biologischer Vorgänge im Pelagial der Helgoländer Bucht. Gesetzmäßigkeiten der Veränderung von Zusammensetzung und Biomasse des Planktons im Jahresgang können bei großer Anzahl von Messungen auf einem Netz von Stationen erkannt werden (HAGMEIER, im Druck). Damit werden jedoch nur mittlere langfristige Verän-

* Herrn Prof. Dr. J. KREY zum 60. Geburtstag in Verehrung gewidmet. 
derungen untersucht. Über Schwankungen der Zelldichte und die Abfolge von Arten in einer bestimmten Planktonbevölkerung hingegen kann nur etwas ausgesagt werden, wenn es gelingt, einen driftenden Wasserkörper als den Lebensraum einer Planktongesellschaft zu verfolgen und in ihm zu messen. Die Möglichkeit des Erkennens kurzzeitiger Veränderungen des Phytoplanktongehalts durch Wachstum, Gefressenwerden und Absinken ist abhängig von der Zeitdauer der Identität eines Wasserkörpers (vgl. p. 390). Die Komplikationen durch die Prozesse der Vermischung verschiedener Wasserkörper und der horizontalen Diffusion sind dabei gerade im gezeitenreichen Flachmeer außerordentlich groß. So hat die einfachere hydrographische Situation in Seen zum Vorsprung der Limnologie bei Untersuchungen der Populationsdynamik and der Okosystemanalyse in situ geführt.

In der vorliegenden Mitteilung wird über erste orientierende Versuche berichtet, kurzzeitige Veränderungen des Partikelgehalts, besonders des Phytoplanktons, zusammen mit Salzgehalts- und Temperaturschwankungen auf drei "Driftstationen“ $z u$ messen. Diese wurden in drei hydrographisch unterschiedlichen Gebieten in der Helgoländer Bucht durchgeführt (Abb. 1). Uber 4-8 Stunden wurden Wassermassen verfolgt, die mit einem Drifkörper markiert waren. In etwa halbstündigen Zeitabständen wurden dabei Wasserproben aus drei verschiedenen Tiefen entnommen. Je eine Driftstation lag vor der Elbmündung (Tonne „Großvogelsand-W" $W^{\prime \prime}$ und vor der Eidermündung (Tonne "Außeneider"). In der Zeit dieser Untersuchungen (August 1968) wurde die Wucherung eines nackten Dinoflagellaten (HickEi et al. 1971) bei auch sonst reichhaltigem Planktonvorkommen in der Helgoländer Bucht beobachtet. Die Messungen bei. Helgoland (Reede) fanden im November 1968 statt; das Wasser enthielt hier nur einen sehr geringen Planktonbestand. Die Positionen der Probenentnahme sind aus Abbildung 1 zu ersehen.

\section{METHODE}

Als Driftkörper zur Markierung der zu verfolgenden Wassermasse wurde ein Kreuz aus zwei aufeinander senkrecht stehenden Holzplatten von je $2 \mathrm{~m}^{2}$ Fläche angefertigt. Das beschwerte Driftkreuz wurde in $5 \mathrm{~m}$ Tiefe (Station "Helgoland": $3,5 \mathrm{~m}$ ) ausgebracht, indem es durch eine dünne Leine entsprechender Länge mit einer kleinen Oberflächenboje verbunden wurde. Diese Boje wurde auf Sicht verfolgt und in ihrer Nähe Schöpfproben in 0,5,5 und $10 \mathrm{~m}$ Tiefe („Helgoland“: $10-20 \mathrm{~m}$ ) wiederholt entnommen. Der Salzgehalt wurde mit dem Salinometer, die Wassertemperatur mit Umkippthermometern bestimmt. Die Trübungsmessung erfolgte an geschöpttem Wasser im Photometer ZeIss Elko II bei $750 \mathrm{~nm}$ (Filter S 75, 5-cm-Küvette); die Werte wurden als Differenz zu reinem Wasser als $\triangle \mathrm{EK} / \mathrm{m}$ errechnet. Das Seston wurde durch gewichtsgleiche Mrtlipore AA Filterpaare $(0,8 \mu \mathrm{m}$ mittlerer Porendurchmesser) abfiltriert, sein Trockengewicht nach drei Stunden Trocknung bei $75^{\circ} \mathrm{C}$ bestimmt. Menge und Zusammensetzung des Phytoplanktons wurde an Schöpfproben mit der Sedimentationstechnik ermittelt. Die Sichttiefe wurde mit der Secchischeibe gemessen. 


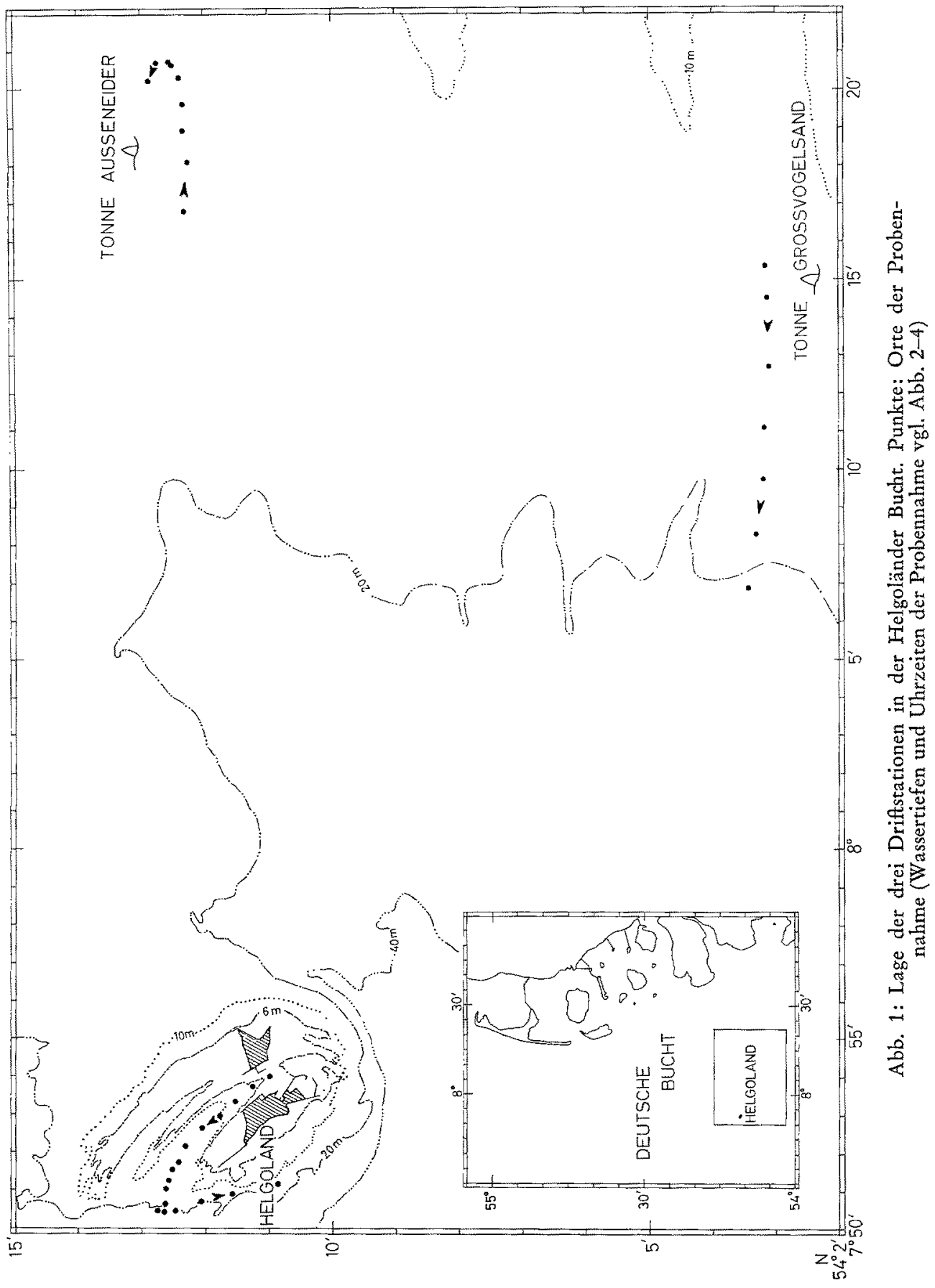




\section{ERGEBNISSE}

$$
\text { Station "Großvogelsand" }
$$

Am 6. August 1968 wurde zwei Stunden nach Hochwasser mit den Untersuchungen im bereits stark ablaufenden Wasser in der Nähe der Tonne "Großvogelsand$W^{\text {“ }}$ vor der Elbmündung begonnen. Die Veränderung von Salzgehalt, Temperatur, Trübung, Sestongewicht und der Konzentration der häufigsten Phytoplankter sind aus Abbildung 2 zu ersehen, in der auch Stromgeschwindigkeit und Wassertiefe angegeben sind. Die Sichttiefe nahm von $2,80 \mathrm{~m}$ zu Beginn auf $2,40 \mathrm{~m}$ gegen Ende der Beobachtungen $a b$. Eine Salzgehalts- und Trübungsschichtung war ausgeprägt. Der stark schwankende Salzgehalt in $10 \mathrm{~m}$ Tiefe ließ eine wechselnde Tiefenlage der Sprungschicht vermuten.

\section{Station "Außeneider"}

Auf dieser Station wurde das auflaufende Wasser untersucht (Abb. 3). Am 8. August 1968 begannen die Messungen südlich der Tonne „Außeneider" um 9.15 Uhr, 2 Stunden 42 min nach Niedrigwasser, und dauerten über die Hochwasserzeit (12.21 Uhr) an. Die Wassersäule war ungeschichtet; der Salzgehalt veränderte sich während der Arbeiten kaum, jedoch wurde das Oberflächenwasser in dieser Zeit um $0,5^{\circ} \mathrm{C}$ erwärmt. Die Sichttiefe schwankte zwischen 3,60 und $4,20 \mathrm{~m}$. Am 6. und 8 . August wehten schwache östliche (ablandige) Winde, die keinen wesentlichen Einfluß auf die Strömungsverhältnisse haben konnten.

Das zur Eigenbewegung befähigte Phytoplankton unterlag im Laufe der Messungen auf beiden Stationen stärkeren Konzentrationsschwankungen in der Wassersäule als das unbewegliche Diatomeenplankton. Besonders auf der Station "Großvogelsand “ fiel eine starke und wechselnde Vertikalschichtung des hier dominierenden nackten Dinoflagellaten* auf, der offenbar in der Lage war, sich trotz der erheblichen Turbu-

\section{Tabelle 1}

Mittelwerte von Seston, Trübung und Diatomeenplankton während der Driftstation „Außeneider". Gemittelt wurden die 3 Messungen einer Vertikalserie, weniger als 3 Werte sind eingeklammert

\begin{tabular}{|cccccc|}
\hline Uhrzeit & $\begin{array}{c}\text { Seston } \\
(\mathrm{mg} / \mathrm{l})\end{array}$ & $\begin{array}{c}\text { Trübung } \\
(\triangle \mathrm{EK} / \mathrm{m})\end{array}$ & $\begin{array}{c}\text { Rhizosolenia } \\
\mathrm{sp.}(50 \mu \mathrm{m}) \\
\left(10^{3} \mathrm{Z} / 1\right)\end{array}$ & $\begin{array}{c}\text { Rbizosolenia } \\
\text { sp. } \\
\left(10^{3} \mathrm{Z} / 1\right)\end{array}$ & $\begin{array}{c}\text { Bodendiato- } \\
\text { meen } \\
\left(10^{3} \mathrm{Z} / \mathrm{l}\right)\end{array}$ \\
\hline 09.55 & 6,6 & 0,244 & 74,7 & 54,4 & 12,8 \\
11.00 & $(4,4)$ & 0,204 & 80,7 & 37,3 & 7,5 \\
12.00 & $(4,1)$ & 0,196 & 94,7 & 35,2 & 12,8 \\
13.10 & 3,2 & 0,189 & 61,3 & 42,7 & 8,5 \\
14.10 & 2,7 & 0,187 & 102,0 & 72,5 & 13,9 \\
\hline
\end{tabular}

* Nach einer Mitteilung von Prof. BraArud, Oslo, handelte es sich nicht um ein Gymnodinitu sp., sondern wahrscheinlich um Gyrodinium aureolum HuLBURT. 


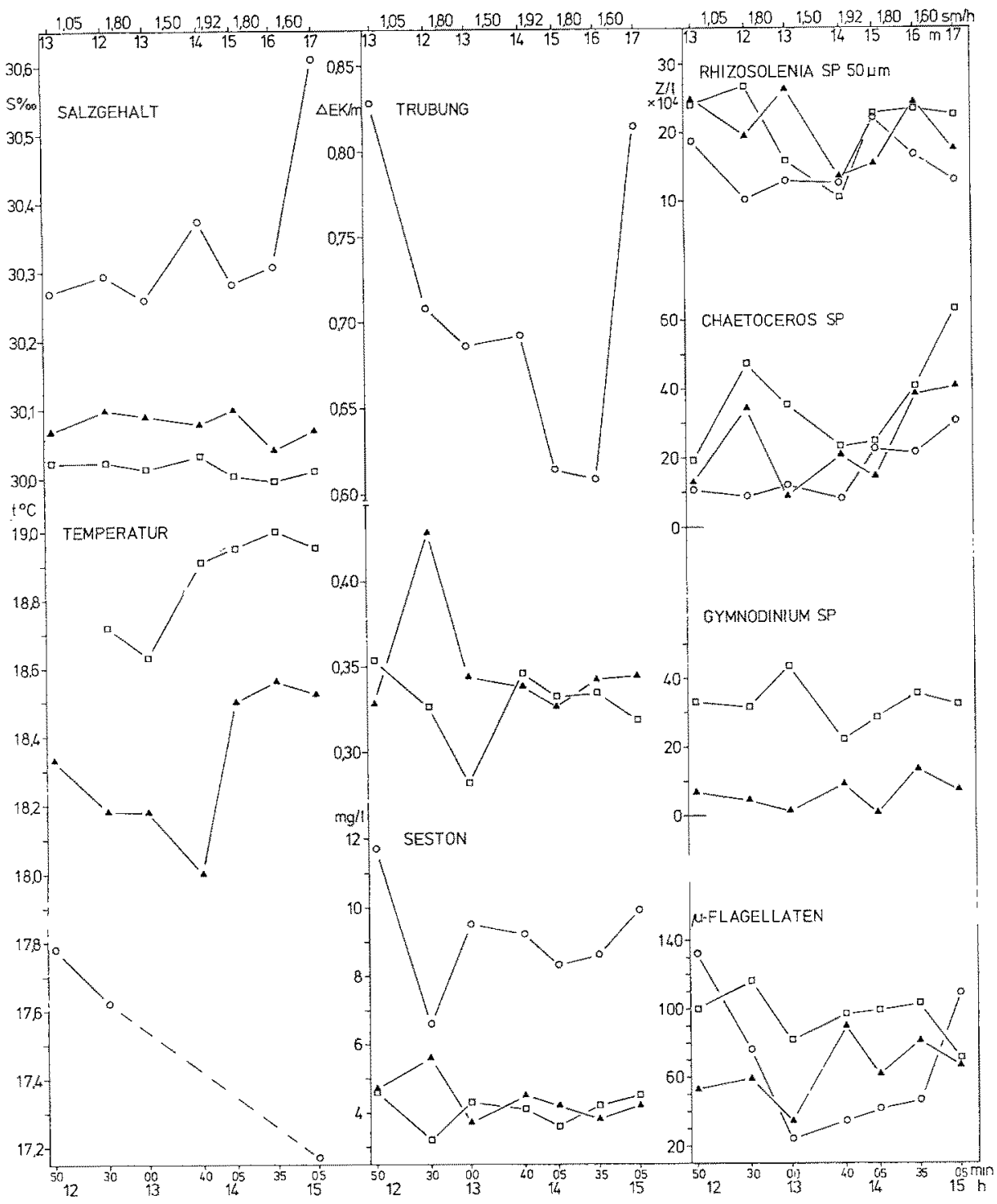

Abb. 2: Driftstation "Großvogelsand“, 6. August 1968. Zeitliche Veränderung von Salzgehalt, Temperatur, Trübung, Sestongewicht und der häufigsten Phytoplankter in den Tiefen $0,5 \mathrm{~m}$ (offene Quadrate), $5 \mathrm{~m}$ (Dreiedse) und $10 \mathrm{~m}$ (offene Kreise). Neben der Uhrzeit der Probennahme (unterer Rand) sind die Strömungsgeschwindigkeiten, die sich aus der Versetzung des Driftkreuzes $z$ wischen den Zeiten der Probennahme ergaben, in $5 m / h$ sowie die gelotete Wassertiefe zur Zeit der Probennahme am oberen Rand der Zeichnung angegeben

lenz in den obersten Metern der Wassersäule anzureichern. In $10 \mathrm{~m}$ Tiefe wurden nur sehr wenige Zellen dieser Art gefunden (Hrckel et al. 1971). Die nach Biomasse dominante Diatomee Rbizosolenia sp. kam mit 100 bis $266 \times 10^{3}$ Zellen/l bei "Groß- 


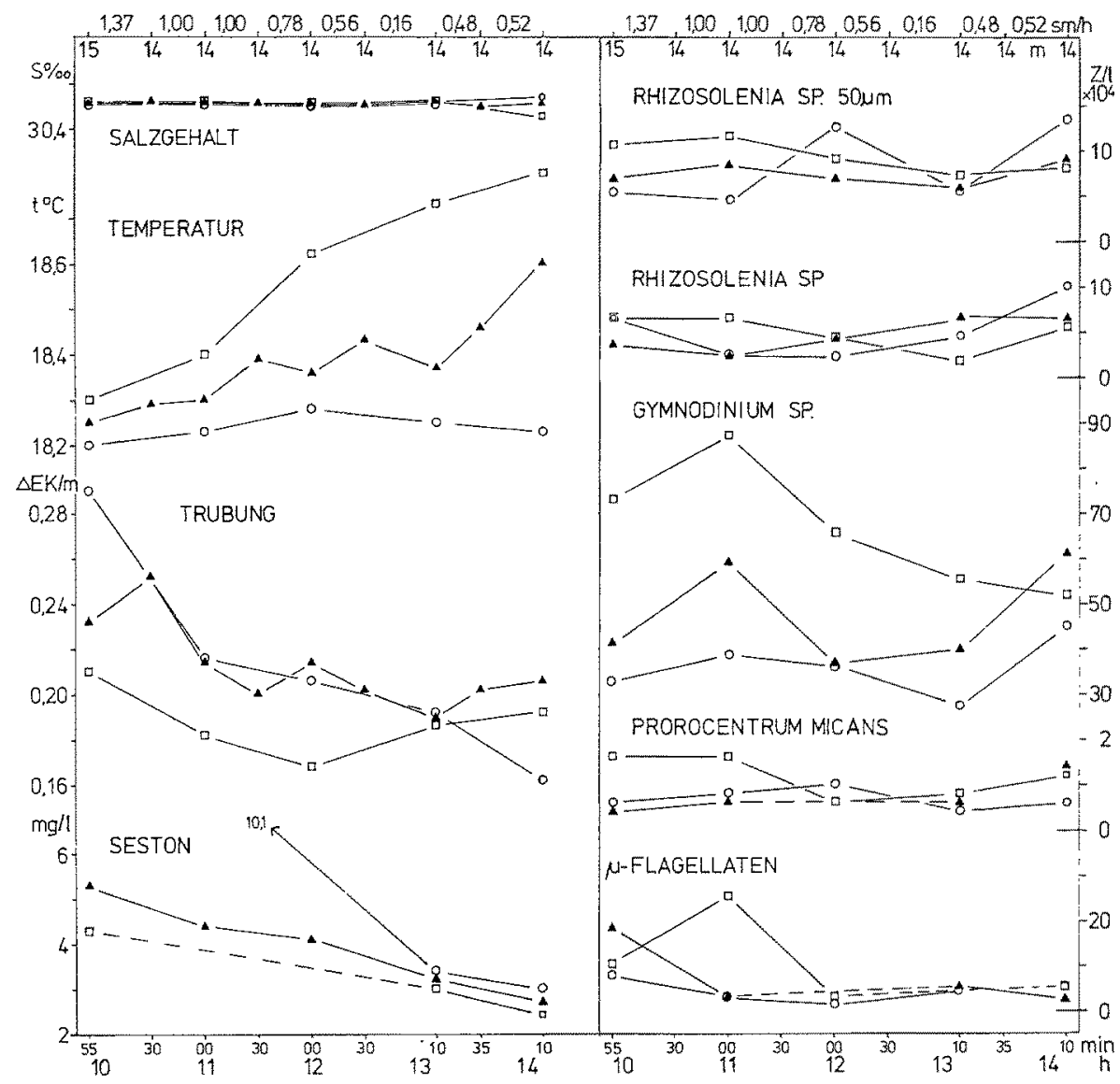

Abb. 3: Driftstation „Außeneider", 8. August 1968. Zeitliche Veränderung von Salzgehalt, Temperatur, Trübung, Sestongewicht und der häufigsten Phytoplankter in $0,5,5$ und $10 \mathrm{~m}$ Tiefe. (Weitere Erklärungen vgl. Abb. 2)

vogelsand" (Mittel $186 \times 10^{3}$ ) und 44 bis $136 \times 10^{3}$ Zellen/l bei "Außeneider" (Mittel $83 \times 10^{3}$ ) vor.

Während das ablaufende Wasser bei „Großvogelsand“ für Untersuchungen der Planktonveränderungen als ungeeignet erscheint, blieb das auflaufende Wasser bei "Außeneider" - nach seinem Salzgehalt zu urteilen - in der Beobachtungszeit gleich und kam für diesen Zweck in Frage. Sestongewicht und Trübung im Wasser sanken hier mit dem Nachlassen des Gezeitenstromes entsprechend der dann schnelleren Sedimentation $a b$ (Abb. 3). Aus einer Zusammenstellung der Mittelwerte aller Tiefen einer Vertikalserie (Tab. 1) ist zu ersehen, daß die nicht beweglichen, lebenden Diatomeenzellen gleichzeitig offenbar keiner verstärkten Sedimentation unterlagen. 


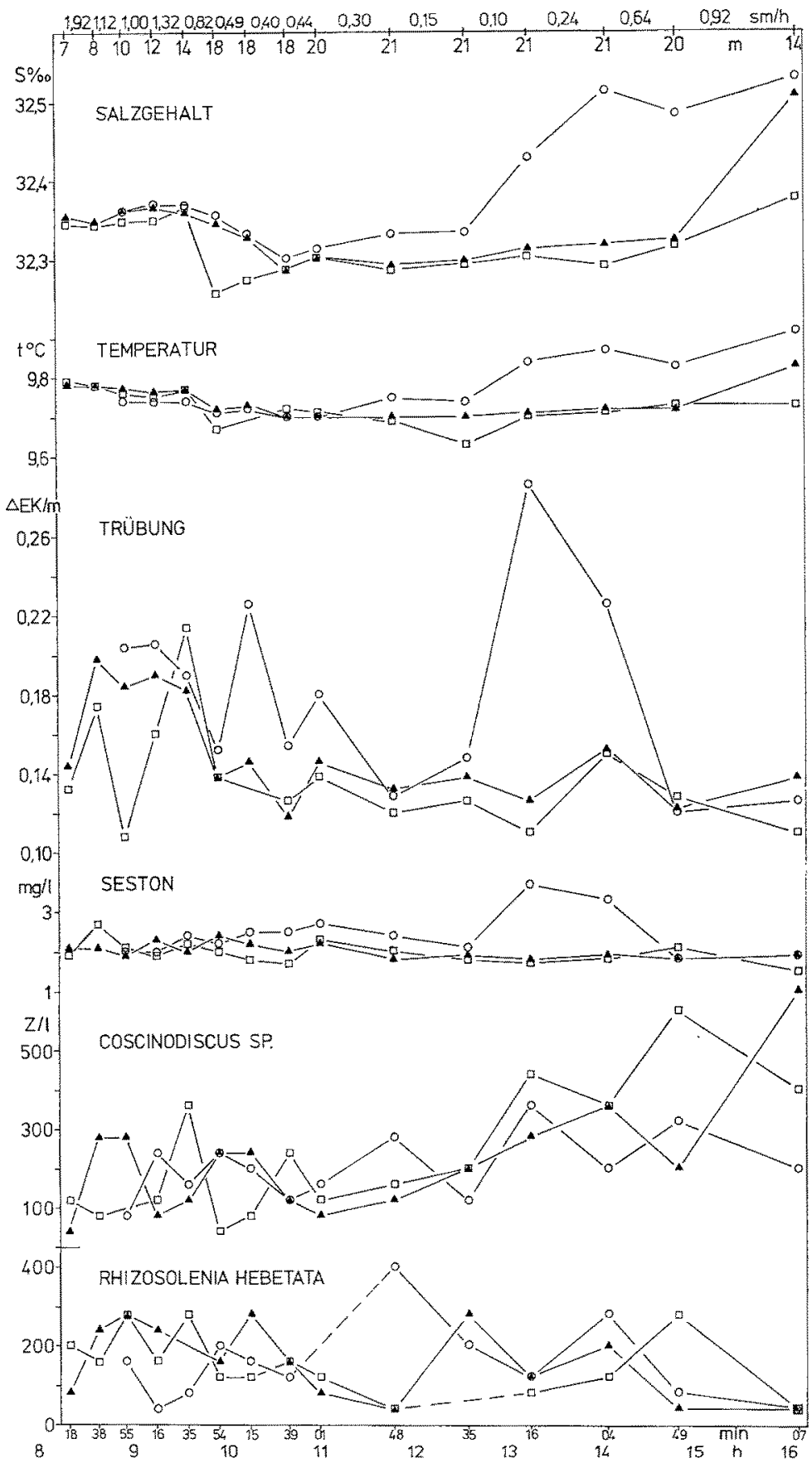

Abb. 4: Driftstation „Helgoland“, 29. November 1968. Zeitliche Veränderung von Salzgehalt, Temperatur, Trübung, Sestongewicht und der häufigsten Phytoplankter in 0,5, 5 und $10-20 \mathrm{~m}$ Tiefe (offene Kreise). (Weitere Erklärungen vgl. Abb. 2) 
Am 29. November 1968 wurde kurze Zeit nach dem Kentern des Stromes (1 h 16 min nach Hochwasser) das Driftkreuz auf der Helgoland-Reede vor dem Dünenhafen ausgesetzt. Bei schwachen, umlaufenden Winden und damit minimaler Beeinflussung des Tidenstromes setzte das Wasser nach Nordosten (Abb. 1). Am Ausgang der Nordreede wurde eine Beimischung salzärmeren Wassers registriert (Abb. 4). Die Tiefenprobe wurde je nach Wassertiefe in 10 bis $20 \mathrm{~m}$ entnommen. Der Strom kenterte nördlich des Repulse-Grundes und lief während der Flut parallel der Südwestseite der Insel auf. Die Beobachtungen wurden um 16.07 Uhr, 2 Stunden nach Niedrigwasser, abgebrochen. Aus den "Karten der Strömungen in der Nähe von Helgoland “ (1929), die ebenfalls durch Messungen mit Drifkreuzen gewonnen wurden, geht hervor, daß der Strom noch etwa 2 Stunden weiter nach Südost und Ost gelaufen wäre. Nach diesen Angaben wäre der hier beschriebene Wasserkörper dann auf der Helgoländer Südreede, ganz in der Nähe des Ausgangsortes, angelangt. $\mathrm{Da}$ von dort das Wasser nach dem Kentern des Stromes wieder nordwestwärts durch die Reede abläuft, muß es als möglich angesehen werden, daß Wassermassen mehrmals um die Insel kreisen können. Dies hat Bedeutung für die Interpretation der seit vielen Jahren durchgeführten Untersuchungen über die Hydrographie und das Plankton der HelgolandReede. Die Salzgehaltsschwankungen (Abb. 4) zeigen, daß das driftende Wasser nicht einheitlich blieb. Allein aus diesem Grund sind die erheblichen Schwankungen der Diatomeenkonzentration nicht zu interpretieren.

\section{DISKUSSION}

Die Existenz verschiedener Wasserkörper in der Inneren Deutschen Bucht kann man beim Uberfliegen des Gebiets bereits ohne Hilfsmittel erkennen. Die Wassermassen besonders im Elbmündungsgebiet, doch oft auch bis wenige Seemeilen südlich von Helgoland, können von unterschiedlicher Färbung sein und sind häufig scharf voneinander abgegrenzt. Beispiele für die hydrographischen, chemischen und biologischen Unterschiede solcher Wassermassen wurden von KALLE (1956) beschrieben.

Die Wassermassen verlieren ihre Individualität durch Vermischungsvorgänge. Das Wasser befindet sich in dauernder turbulenter Bewegung, die durch Gezeitenstrom, winderzeugte Strömungen und Dichteunterschiede bedingt sind. Den Diffusionserscheinungen durch turbulente Wasserbewegung unterliegt auch die Verteilung des Planktons, besonders die der unbeweglichen Formen. Welche Größenordnung die horizontale Diffusion im Meere hat, geht aus Arbeiten von Stommel (1949), JosepH \& SENDNER (1958) und Joseph et al. (1964) hervor. Joseph et al. konnten zeigen, wie sich eine Farbstoffwolke in verschiedenen Gebieten der Nordsee im Laufe von einigen Tagen durch Diffusion ausbreitete. Die Autoren brachten in einer Reihe von Versuchen in der Deutschen Bucht und der Mittleren Nordsee große Mengen (bis $450 \mathrm{~kg}$ ) des Farbstoffs Rhodamin b punktförmig aus und maßen dessen Verdünnung fluorometrisch. Die Versuche dauerten zwischen 69 und 114 Stunden. In dieser Zeit erreichte die Rhodaminausbreitung eine Flächenausdehnung von etwa 25 bis $55 \mathrm{~km}^{2}$. 
Bereits in den ersten 24 Stunden war der Farbstoff von der Oberfläche bis in $20 \mathrm{~m}$ Tiefe gleichmäßig verteilt, wo eine schwache Sprungschicht eine weitere Ausbreitung verhinderte. Die wahrscheinlichsten Diffusionsgeschwindigkeiten für die genannten Seegebiete wurden mit 0,2 bis $0,4 \mathrm{~cm} / \mathrm{sec}$ errechnet.

In den von uns untersuchten Wasserkörpern boten sich nur in einem Falle (Driftstation "Außeneider") die Voraussetzungen für eine Untersuchung kurzfristiger Veränderungen des Partikelgehalts, da Anderungen des Salzgehalts bei den anderen Stationen Zumischung andersartigen Wassers erkennen ließen. Bei der Station „Außeneider" waren die gewichtsmäßig dominierenden Partikel wie auch die kleinen Partikel $(<10 \mu \mathrm{m} \phi)$, welche iberwiegend die Trübung verursachen, nicht aber die lebenden Diatomeen in ihrer Konzentration von der Gezeitenstromgeschwindigkeit abhängig.

In den hier gewähiten kurzen Untersuchungszeitspannen von 4 bis 8 Stunden sind Anderungen des Partikelgehalts im Wasser vor allem durch Gezeitenstrom-Schwankungen zu erwarten. Untersuchungen biologischer Veränderungen der Planktondichte, etwa durch Wachstumsprozesse, haben vor allem die Teilungsrate der Phytoplankter (maximal ein- bis zweimal pro Tag) in Rechnung zu stellen, sind also über mehrere Tage fortzuführen. Dafür muß nicht nur die Verdriftung, sondern auch die Art der Veränderung des Wasserkörpers durch Vermischung und Diffusion bekannt sein. Im Hinblick auf dieses Problem kann es sich bei den hier beschriebenen Messungen nur um eine Prüfung der Eignung verschiedener Wassermassen handeln.

Die Messung der Veränderung in einer Planktonbevölkerung der Helgoländer Bucht würde zunächst die Untersuchung der Größe und Abgrenzung der Wasserkörper voraussetzen. Ferner wäre die Heterogenität der Planktonverteilung im als geeignet befundenen Wasserkörper festzustellen, nach der sich die Genauigkeit der quantitativen Planktonzählung zu richten hat (GILLBRIchT 1962). Schließlich wird neben der Verfolgung des Driftweges die umfangreichste Arbeit darin bestehen, die Diffusionsgeschwindigkeit, etwa durch Farbstoffyerdünnung, zu messen und sie rechnerisch für die gefundene Partikelveränderung zu berïcksichtigen. So würde allein die Messung des Phytoplanktonwachstums in situ über mehrere Tage hinweg eine aufwendige Gemeinschaftsarbeit mit möglichst mehreren Schiffen bedeuten, was den Einsatz einer besonders leistungsfähigen Arbeitsgruppe erfordert.

\section{ZUSAMMENFASSUNG}

1. In der Helgoländer Bucht wurden vor der Elbe- und Eidermündung sowie bei Helgoland driftende Wassermassen 4-8 Stunden lang untersucht. Dabei wurden in etwa halbstündigen Zeitabständen Vertikalserien von Wasserproben entnommen und Salzgehalt, Temperatur, Trübung und Sestongewicht bestimmt sowie Phytoplankton gezählt (Abb. 2-4).

2. Lediglich das auflaufende Wasser bei der Driftstation „Außeneider" blieb hydrographisch unverändert; nur hier waren die Voraussetzungen für die Messung kurzzeitiger Veränderungen des Partikelgehalts gegeben. Das unbewegliche Plankton 
(Diatomeen) zeigte nicht den raschen Abfall der Konzentration mit dem Nachlassen des Gezeitenstroms wie das Sestongewicht und die Trübung.

3. Die Verfolgung driftenden Wassers bei Helgoland ließ erkennen, daß durch die besonderen Gezeitenstromverhältnisse Wassermassen mehrmals um die Insel kreisen und die Helgoland-Reede passieren können.

4. Diese Untersuchungen wie auch veröffentlichte Ergebnisse von Messungen der horizontalen Diffusion im Meere zeigen, daß Untersuchungen der Veränderungen innerhalb einer einzelnen Planktonpopulation im Laufe von mehreren Tagen in der Helgoländer Bucht eine aufwendige Gemeinschaftsarbeit erfordern.

Danksagung. Frau A. Reiners und Herrn K. Treutner sei für ihre sorgfältige Mitarbeit gedankt.

\section{ZITIERTE LITERATUR}

Grltbricht, M., 1962. Über das Auszählen yon Planktonschöpfproben. Helgoländer wiss. Meeresunters. 8, 203-218.

Hagmerer, E. Coastal influence on microplankton in Heligoland Bay (North Sea). (6th Europ. Symp. Marine Biology, Rovinj.) Thalassia jugosl. (In press).

Hrckel, W., HAGMeIER, E. \& DreBes, G., 1971. Gymnodinium blooms in the Helgoland Bight (North Sea) during August, 1968. Helgoländer wiss. Meeresunters. 22, 401-416.

Joseph, J. \& SENDNER, H., 1958. Über die horizontale Diffusion im Meere. Dt. hydrogr. Z. 11, 49-77.

Joserh, J., SENDNer, H. \& WeIdemann, H., 1964. Untersuchungen über die horizontale Diffusion in der Nordsee. Dt. hydrogr. Z. 17, 57-75.

KALLE, K., 1956. Chemisch-hydrographische Untersuchungen in der Inneren Deutschen Bucht. Dt. hydrogr. Z. 9, 55-65.

Karten DER Strömungen in der Nähe von Helgoland. 1929. Veröff. Marineobs. Wilhelmsh. Srommel, H., 1949. Horizontal diffusion due to oceanic turbulence. J. mar. Res. 8, 199-225.

Anschrift des Autors: Dr. W. Hickel

Biologische Anstalt Helgoland (Zentrale)

2 Hamburg 50

Palmaille 9

Bundesrepublik Deurschland 